\title{
LOS ORÍGENES DEL SANCHEZCERRISMO EN TACNA (1931-1934)
}

\author{
SANCHEZCERRISMO'S ORIGINS IN TACNA (1931 - 1934)
}

Alfonso Renato Vargas Murillo

\section{RESUMEN}

El Partido Unión Revolucionaria, si bien no tuvo una presencia masiva en la localidad, como si lo tuvo el APRA, fue en la década de los 30 un movimiento importante, monopolizó la opinión pública por medio de sus órganos de expresión (como el diario "La Verdad"), esto como producto de la censura decretada por el gobierno a la oposición, representada principalmente por el aprismo, lo que desencadenó una serie de conflictos en la capital y en el interior del país, que dejó como saldo víctimas mortales (civiles y militares), entre ellos, el propio Presidente de la República. En Tacna, ubicamos la presencia del "sanchezcerrismo", representado en un inicio por los "Clubes Sánchez Cerro" y en un segundo momento por el Partido Unión Revolucionaria, visualizado a través de sus órganos de expresión, como: "La Nación", bajo la dirección de Augusto Villa de la Tapia, como diario adherente a la "Revolución de Arequipa" y furibundamente anti-aprista, así como en "La Verdad”, órgano propagandístico del partido de Sánchez Cerro.

Palabras clave: APRA, Sánchez Cerro, Unión Revolucionaria.

\section{ABSTRACT}

The Revolutionary Union Party; although, it did not have a massive presence in the locality, as APRA had, was in the decade of the 30 an important movement that monopolized the public opinion through its organs of expression (as " The Truth " Newspaper). This happened as a result of the censorship decreed by the government to the opposition, represented mainly by the aprismo, which triggered a series of conflicts in the capital and in the interior of the country, leaving as a result mortal victims (civilians and military), including the President of the Republic. In Tacna, the presence of the "sanchezcerrismo" was located, represented at first by the "Sanchez Cerro Clubs" and in a second moment by the Union Revolutionary Party, visualized through its expression organs, such as: "The Nation", under the direction of Augusto Villa de la Tapia, as a daily adherent to the "Revolution of Arequipa" and fiercely antiAPRA, as well as in "La Verdad", propagandistic organ of the Sánchez Cerro party.

Keywords: APRA, Sánchez Cerro, Revolutionary Union

\section{INTRODUCCIÓN}

La tercera década del siglo pasado se inicia con la caída de Augusto B. Leguía, el gobierno de la junta militar y el retorno de Tacna al seno patrio buscando recuperarse de los traumáticos sucesos del cautiverio. Esta década también se distingue por el surgimiento de nuevas fuerzas políticas -como el aprismo y el socialismo- la resistencia y oposición de una derecha debilitada en ese entonces y fuerzas nacionalistas que virarían al fascismo, teniendo como máximo exponente al partido Unión Revolucionaria, fundado por Luis M. Sánchez Cerro. La violencia política desencadenada, culminaría con el asesinato del Presidente por un militante aprista y en el plano internacional las relaciones con el Eje que se consolidan con Oscar R. Benavides; es así como se caracterizaría este periodo de profundas contradicciones en todos los campos.

En el plano local, trabajos como los de Oscar Panty Neyra, en su obra "Historia de la Prensa Escrita en Tacna" y "Función Orientadora de la Prensa Escrita", nos brindan una visión general de los diversos diarios de la época y su tendencia, así como la función que cumplieron conforme se desarrollaban los acontecimientos políticos nacionales. Así podemos reconocer para nuestro estudio a los diarios "La Nación" y "La Verdad"; el primero por ser testigo de debates y ataques entre el aprismo y la incipiente oposición sanchezcerrista y el

\footnotetext{
${ }^{1}$ Estudiante de la Facultad de Educación, Comunicación y Humanidades, Escuela Profesional de Historia, Universidad Nacional Jorge Basadre Grohmann y de la Facultad de Derecho de la Universidad Privada. Tacna-Perú. E-mail: avargasm95@gmail.com
} 
segundo por ser órgano de expresión de este último hasta el fallecimiento de su líder Luis Miguel Sánchez Cerro.

Sobre la historia del Partido Unión Revolucionaria, trabajos como "El Pensamiento Fascista", de José Ignacio López Soria y "El Fascismo en el Perú", de Tirso Molinari Morales, son elementos clave debido a que recopilan un gran número de fuentes que nos permiten observar la evolución del partido y su extensión en el plano nacional, pero en los cuales poco o nada se ha podido hallar sobre la presencia en Tacna. Este primer trabajo pretende llenar ese vacío historiográfico sobre la presencia del sanchezcerrismo en la localidad dentro de la agitada coyuntura de los años 30, respondiendo a las siguientes preguntas:

¿Existió un movimiento de carácter sanchezcerrista en Tacna?, de ser afirmativa la respuesta, ¿De qué manera se organizó? y ¿Qué papel ocupó en el contexto político post-cautiverio en Tacna?

\section{El Partido Unión Revolucionaria}

El Partido Unión Revolucionaria tuvo como antecedente al "Comité de Saneamiento y Consolidación Revolucionaria”, creado durante el primer gobierno transitorio de Luis M. Sánchez Cerro en enero de 1931, el cual sirvió de nexo organizativo para la creación del partido por disposición del mismo, así como los "Clubes Sánchez Cerro" creados espontáneamente en la capital y el interior del país. Así, el PUR se funda el 30 de julio de 1931, estructurándose de manera vertical y jerarquizada, que iba desde el comité nacional representado por el Comité Central Directivo, hasta los comités locales representados por los Comité Departamentales y provinciales (Molinari, 2009, p. 46).

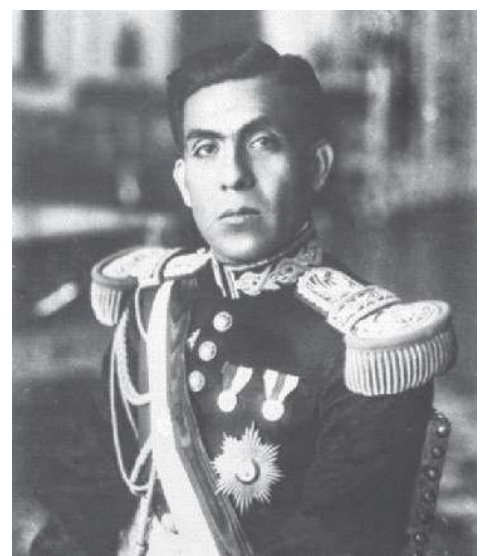

Figura 1: Retrato de Luis M. Sánchez Cerro [fotografía] Fuente: http://repositorio.pucp.edu.pe/index/handle/123456789/9833
Para entender el fenómeno del urrismo, desde su origen alrededor del caudillo Sánchez Cerro y su transformación en un partido de corte fascista, es necesario diferenciar ambos fenómenos como dos momentos de la Unión Revolucionaria. Para tal fin, es necesario hacer un análisis comparativo, como el que realiza Stanley G. Payne en "Entre los movimientos fascistas per se y la derecha autoritaria no fascista (o a veces protofascista)", basándonos en la tipología de Ernst Nolte, con los aportes de J. Puhle: el anti-marxismo, antiliberalismo, anti-conservadurismo (en lo político), el principio del caudillaje, un ejército del partido, el objetivo del totalitarismo. Dentro de esta tipología, los tres primeros puntos resultan "negaciones fascistas" como los llama Payne y el resto características que podríamos denominar partidarias. Pero a estas características, J. Puhle, decide agregar la doctrina económica, que es el corporativismo (Payne, 2005, pág. 12). Franco Savarino, realiza una descripción general basada en los rasgos particulares del fascismo:

[...] el liderazgo carismático, la movilización de masas, la hostilidad hacia la democracia, el liberalismo y el marxismo, el rechazo de los valores burgueses, el ethos heroico y romántico, el culto y los rituales "civiles", la tendencia mitopoietica, la exaltación de la virilidad, de la juventud, del superhombre "nietzscheano", la valoración positiva de la violencia y de la guerra, el glamur estético, el elitismo, el "holismo" jerárquico, el voluntarismo, el vitalismo, el idealismo metafísico y el impulso hacia la trascendencia (Savarino, 2005, p. 264).

Ambas tipologías, buscan evitar análisis del tipo reduccionistas. En base a las características señaladas por los diversos autores y con la evidencia documental que cita Tirso Molinari, podemos denominar como un partido de corte fascista a la Unión Revolucionaria en su segundo momento al mando de Luis A. Flores. Es así que, el primer momento, gira en torno al carisma y el culto a la personalidad de Sánchez Cerro, siendo el eje articulador de un movimiento heterogéneo de carácter populistaconservador (Molinari, 2006, p. 323).

El primer momento parte de la Revolución de Arequipa, la toma de poder por la junta de gobierno, el apoyo masivo hacia la gesta de Sánchez Cerro, que en un inicio se agrupa en "Clubes Sanchezcerristas" y emprende el camino hacia lograr un sanchezcerrismo orgánico, es así que se funda el Partido Unión Revolucionaria. Tanto en el programa de gobierno como en el accionar del mismo, podemos considerar al urrismo durante el primer momento como proto-fascista. Es así que el sanchezcerrismo "sigue la línea de un populismo «de derecha» de tintes nacionalistas autoritarios, a caballo 
entre el militarismo conservador tradicional y una cierta mimesis fascista" (Gonzales, 1994, p. 230).

Una visión general de la Unión Revolucionaria, la ofrece Gonzales Calleja, pero es necesario dividirla para ilustrar mejor ambos momentos:

\begin{abstract}
El movimiento sanchezcerrista presenta de forma paradigmática los rasgos contradictorios del populismo 'de derecha': retórica revolucionaria centrada en lo político (el antileguiismo era una de sus señas de identidad más patentes); conservadurismo primario evidenciado en el rechazo de los partidos tradicionales y de los movimientos e ideas 'disolventes'; nacionalismo popular regeneracionista y moralizador en principio, con exaltados tintes xenófobos simbolizados en el slogan urrista «Perú para los peruanos»; liderazgo carismático de militarismo romántico; personalismo y paternalismo en la gestión de los problemas[... ] (Gonzales, 1994, p. 233).
\end{abstract}

Hasta aquí el primer momento "sanchezcerrista", donde se puede observar un "populismo conservador y carismático" (Molinari, 2006, p. 322), bajo el liderazgo de Sánchez Cerro.

[... ]visión corporativista de la política, de la sociedad y del Estado; afirmación de los valores espirituales (defensa de la religión, de la moralidad y de la honradez, condensados en el lema «Verdad, justicia, integridad, patriotismo»), una idea del progreso nacional conciliable con la defensa de la propiedad privada y dirigido a la renovación y modernización de las estructuras socioeconómicas tradicionales, (equilibrio entre el capital y el trabajo, desarrollismo económico en pro del bienestar social, fomento de la educación, descentralización administrativa, saneamiento financiero (...) pero no a la introducción de nuevas relaciones de producción (Gonzales, 1994, p. 233).

En una segunda etapa, como se mencionó antes, el proyecto del Estado corporativo no fue asumido en un inicio, sino hasta la muerte del caudillo y la asunción de la jefatura por Luis A. Flores, donde se transforma en un partido fascista. Por lo tanto, podemos afirmar que las "negaciones fascistas", como las denomina Payne (antimarxismo, antiliberalismo, anticonservadurismo), son asumidas plenamente por el partido Unión Revolucionaria en su etapa fascista, no así en un inicio, existiendo tolerancia con el demo-liberalismo y los sectores conservadores oligárquicos, por la composición heterogénea del partido, donde coexistían -como señala Tirso Molinari- "sanchezcerristas viscerales, antileguiistas, demoliberales, neocivilistas y neoindigenistas" (Molinari, 2009, p. 39).

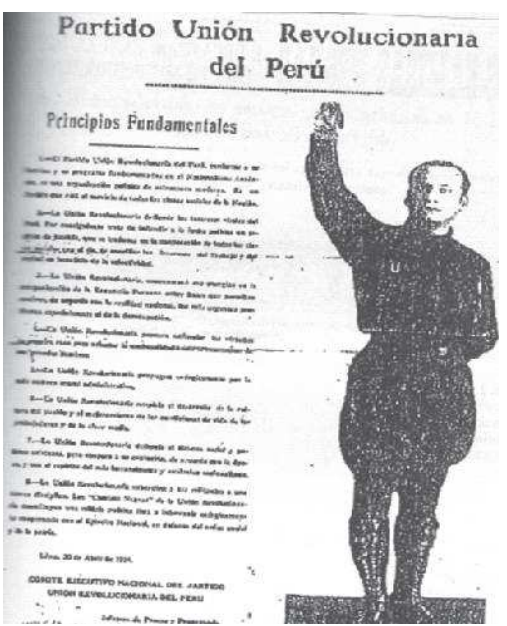

Figura 2. Volante de la UR de 1934. Luis A. Flores, líder urrista. Fuente: Molinari T. (2009)

El "principio del caudillaje" -que nace con el sanchezcerrismo, el culto mesiánico al líder- se mantiene con Luis A. Flores como Jefe Supremo y Sánchez Cerro como "líder espiritual". El ejército del partido se vio materializado en la legión de camisas negras, tomando la mística y estética del Partito Nazionale Fascista italiano. El Estado totalitario, materializado en el Estado corporativo, donde se articulan orgánicamente las "fuerzas vivas" de la Nación, como decía Mussolini: "Todo en el Estado, nada contra el Estado, nada fuera del Estado" (Mussolini, 1940, p. 144). Esta última característica quedó en el plano teórico después de la muerte de Sánchez Cerro, pues en su gobierno (a pesar del carácter autoritario con la que enfrentó la convulsionada situación político-social) no se realizaron cambios estructurales orientados hacia un modelo de Estado corporativo; este proyecto fue asumido por Luis A. Flores y el urrismo en su etapa fascista, pero nunca se pudo plasmar al no poder capturar el poder político (Molinari, 2006, p. 324).

Y por último, la visión antropológica del "nuevo hombre del fascismo" o el "hombre trágico" (López, 1981, p. 9) será fácilmente observable en esta segunda etapa del urrismo: el culto mesiánico y la exaltación del heroísmo y las cualidades del caudillo Sánchez Cerro dentro del intento de mantener el liderazgo carismático luego de su muerte, la entrega absoluta a un ideal suprahumano como la patria, la férrea disciplina y el combate asumido como una lucha espiritual que se tradujo en una violencia física y simbólica en pos de la "civilización contra la barbarie" (López, 1981, p. 10) que, según esta visión, representaban las fuerzas apristas y comunistas. 
Este trabajo se enfocará en el primer momento del Partido Unión Revolucionaria hasta el punto de quiebre, donde asumirá el liderazgo Luis A. Flores, quien será el artífice del viraje hacia el fascismo de modelo italiano.

\section{La Unión Revolucionaria y el periodismo}

En el plano nacional, diversos periódicos servían como medios de difusión del sanchezcerrismo y posteriormente al urrismo en sus dos etapas. Como señala López Soria, el primer diario fue "La Opinión", órgano del sanchezcerrismo, perteneciente a esta primera etapa. En la segunda etapa, estuvieron "Crisol" (órgano de las camisas negras), "Acción” y "La Batalla”. (López, 1981, p. 25) En un inicio, la función del periodismo sanchezcerrista se basó en realzar la imagen y la gesta del líder carismático, luego sirvió para canalizar el apoyo popular y el culto mesiánico de manera orgánica y vertical hacia Sánchez Cerro, lo cual se tradujo en la formación del Partido Unión Revolucionaria y es cuando la prensa adquiere del mismo modo un carácter orgánico. Entendiendo al periodista como un actor social, sujeto a una serie de condicionamientos, con determinado interés, clase y grupo social, actuando de esta manera, como "portavoz de la ideología dominante" (Morán y Aguirre, 2008 , p. 238), se puede inferir, que aunque determinados diarios no se presentaron a sí mismos como órganos del expresión del partido, hubieron una serie de diarios que se alinearon al régimen, por intereses de toda índole, sirviendo para la consecución de sus fines.

Es así que, durante la campaña electoral y el régimen del Sánchez Cerro, la labor de estos diarios fue vital, en cuanto cumplía dos funciones principalmente, -en terminología de Maxwell E. Mccombs-, "reforzar y cristalizar". La primera resulta en: "consolidar actitudes y opiniones ya existentes...facilita la evidencia y tal vez el apoyo social para respaldar una postura ya asumida por el votante" (McCombs, 1994, p. 99). Es decir, en esta función, se buscaba aprovechar ciertas condiciones culturales, enraizadas en la población, para conducir el apoyo hacia el caudillo -como lo explica Molinari- caracterizándose el Perú de la década de 1930, por lo siguiente:

[...] fuertes rasgos tradicionales-autoritarios de la vida social en el país ... basada estructuralmente en un sistema político neopatrimonialista y en el poder oligárquicolatifundista en crisis, así como instituciones familiares, eclesiásticas y laborales bastante verticales y en relaciones de género muy asimétricas (Molinari, 2009, p. 19).

La segunda se refiere a "la evolución o aprendizaje de una actitud. En su extremo significa adquisición de una actitud cuando previamente no existía ninguna, pero con mayor frecuencia quiere decir reforzar y dar forma a alguna vaga predilección anterior" (McCombs, 1994, p. 99). Esta segunda función puede explicar como el sanchezcerrismo en un inicio tuvo una composición tan heterogénea, en cuanto a la clase y tendencia política, que desencadenará posteriores quiebres y rupturas dentro del partido.

Esta composición heterogénea del urrismo y la no identificación del partido como fascista, sivió para que en un inicio, incluso por medio del diario La Opinión, se tacharáal aprismo de fascista y viceversa, con el único fin de descalificar al adversario político -práctica común, equiparable a la reductio ad-hitlerum, de Leo Strauss (1965, pp. 42-43)-, pese a que en esta etapa existía cierta admiración hacia el gobierno de Mussolini por parte de Sánchez Cerro, como señala una carta escrita desde Paris el 8 de abril de 1931, donde -hace alusión al régimen fascista italiano- señala: "tengo varios amigos, funcionarios públicos que me facilitarán que tome nota de algunos puntos importantes de ese país actualmente tan bien organizado" (Molinari, 2009, p. 42).

Sin embargo, al analizar su gobierno, podemos inferir que esto se tradujo en una recepción de los aspectos más conservadores del fascismo, siendo una interpretación pragmática del mismo.

A la muerte trágica de Sánchez Cerro, Luis A. Flores ofrece, como afirma López Soria "un rostro definido, los fascistas de Unión Revolucionaria acuñaron o copiaron himnos, emblemas y gestos, hicieron de la tumba de Sánchez Cerro un lugar de peregrinaje y desfilaron por las calles de Lima luciendo sus "camisas negras"" (López, 1981, p. 26).

En el plano local, la "Unión Revolucionaria" tuvo como máximo órgano propagandístico a "La Verdad". Como antecedente, un breve momento en "La Nación" a cargo de Augusto de la Villa Tapia y los "Clubes Sánchez Cerro". En cuanto a expresiones "fascistizantes" mas no urristas, se pueden visualizar en "El Caplina" y "La Voz de Tacna".

\section{Presencia de la Unión Revolucionaria en la prensa escrita de Tacna}

Oscar Panty Neyra, en su trabajo sobre la "Historia de la Prensa Escrita en Tacna”, en el periodo de 1929-1939, señala que "la prensa fue tomando posición comprometida con los intereses en conflicto, bajo la influencia de las nuevas fuerzas operantes en el país" (Panty, 1998, p. 84). En esta coyuntura se perciben cambios bruscos en las líneas editoriales de los periódicos, ataques que buscan desacreditar y deslegitimar lo dicho por el diario de la 
tendencia opuesta, así como persuadir al lector -realzando el nombre del candidato/caudillo o régimen-y neutralizando violentamente toda tendencia opuesta.

En el mismo trabajo, realiza una clasificación de los diarios de la época por tendencia política, calificando a tres diarios como sanchezcerristas, siendo estos: "La Verdad" (1932-1934), "El Caplina" (II expresión) (1934-1936) y "La Voz de Tacna” (III expresión) (19361939). Cabe recalcar que, dentro de esta clasificación, se puede considerar al diario "La Nación", pero en su "3er momento fascistizante" (Panty, 1998, p. 84), que por la brevedad de su existir bajo esta tendencia, no es considerado. El caso de "El Caplina" como la "La Voz de Tacna", presentaban explícitamente su adhesión al gobierno de Oscar R. Benavides, lo cual, los descalificaría como sanchezcerristas, pues es en ese momento donde se produce un quiebre en la Unión Revolucionaria, siendo considerado el partido de Benavides un partido traidor al proyecto sanchezcerrista y este devuelve el golpe persiguiendo a urristas, como Luis A. Flores, tal como señala Eduardo Gonzales Calleja:

Tras la desaparición de Sánchez Cerro, los sectores oligárquicos se dispusieron a desactivar la amenaza populista mediante la instauración de un régimen militar clásico. Resulta sugestiva la comparación de las maniobras de inhabilitación realizadas por el presidente interino general Benavides respecto al movimiento sanchezcerrista [...]. Al comienzo, se apoyó sobre la UR para mantener el hostilizamiento sobre el PAP, pero en junio de 1933 reorganizó el gabinete excluyendo a Luis A. Flores -entonces ministro de Marina- y cediendo amplias esferas de poder a los viejos civilistas (Gonzales, 1994, p. 236).

Es así que en su obra "Función orientadora en la Prensa Escrita", se les denomina "diarios fascistizantes", explicando que demuestran una "actitud proclive a los gobiernos autoritarios y centralistas y contraria a las alternativas populares de orientación socialdemócrata y marxista” (Panty, 2001, p. 44), por cuestiones prácticas, utilizaremos este término para catalogar los diarios antes mencionados -en el sentido que no representan una adhesión orgánica al fascismo per se-representado en el segundo momento de la Unión Revolucionaria, pero que mantenían una visión positiva sobre los regímenes fascistas en Europa, que se presentó tanto en la prensa sanchezcerrista, como en la benavidista.

\section{Antecedentes del Partido Unión Revolucionaria en Tacna: Los “Clubes Sánchez-Cerro”}

El Partido Unión Revolucionaria tuvo como antecedente el "Comité de Saneamiento y Consolidación Revolucionaria”, que se creó durante su primer gobierno transitorio, en enero de 1931, sirviendo de nexo organizativo para la creación del partido por disposición de Sánchez Cerro, así como los "Clubes Sánchez Cerro" creados espontáneamente en la capital y el interior del país. El PUR (en adelante Partido Unión Revolucionaria) se fundó el 30 de julio de 1931, estructurándose de manera vertical y jerarquizada, que iba desde el comité nacional representado por el "Comité Central Directivo", hasta los comités locales, representados por los Comité Departamentales y provinciales (Molinari, 2009, p. 46). Si bien estos clubes, ocupaban el lugar inferior en la estructura orgánica del partido, fueron determinantes para el triunfo del candidato urrista, en cuanto -como señala Paulo Drinot- permitieron establecer los vínculos entre el partido y las masas" (Drinot, 2001, p. 338).

Es así, que en el plano local, la primera manifestación del sanchezcerrismo como fuerza política, son los Clubes de apoyo a la candidatura de Sánchez Cerro, el "Club Sánchez Cerro N${ }^{\circ}$ ” y el "Club Sánchez Cerro N 2". Si bien el diario "La Nación" no se muestra como órgano propagandístico, deja clara su visión respecto a la denominada "revolución de Arequipa", al señalar que dicho comité "[...] trabajará por el triunfo de la candidatura del militar que derrocó la tiranía de los 11 años". (La Nación, 22 de agosto de 1931, p. 1). Adhiriéndose de esta manera al proyecto sanchezcerrista, el cual se estaba construyendo sobre la base de "La Revolución de Arequipa" como mito político.

La primera expresión mediática y propagandística del incipiente club sanchezcerrista fue el manifiesto del Presidente del "Club Sánchez Cerro $\mathrm{N}^{\circ} 02$ " Arturo F. Zerpa -de apoyo a la candidatura del General- titulado "Candidatura Nacional", en la cual se refleja los rasgos populistas-autoritarios y carismáticos, comunes en los adherentes del incipiente sanchezcerrismo. El presidente del Club expresará:

iComandante Sánchez Cerro! Si tu nombre es un honor para Piura, que te vio nacer, también será un luminoso punto en la historia, la que tu hazaña persistirá modelado con carácter de viva gratitud [... T Tacna no es indiferente, su situación de enorme desventura le impone ecuanimidad, inteligente serenidad, pero llegado el momento esperado ella hará sentir también los afectuosos vivas por el héroe del 22 de agosto, (La Nación, 25 de agosto de 1931, p.1).

A escasos días de la intempestiva salida de Augusto Villa de la Tapia del diario "la Nación", se produce por primera vez de manera explícita la declaración de guerra al 
aprismo y la adhesión a la candidatura del Comandante Sánchez Cerro, en la habitual columna de "El momento Político", titulada esta vez "Peruano antes que nada", donde desde un inicio manifiesta que no hará un análisis de las candidaturas y solo se remitirá a hablar del candidato que "salvará al país del caos", resaltando las cualidades personales y profesionales del Comandante, expresando: "desde que se iniciara en la gloriosa carrera de las armas, destaca entre jóvenes compañeros por su capacidad teórica, amor a su carrera y ascendrado (sic.) Patriotismo, su lema fue siempre por la patria y para la patria”. (La Nación, 13 setiembre 1931, p. 4)

Con motivo de las elecciones del 11 de octubre de 1931 -donde Sánchez Cerro resulta ganador- y al no haber obtenido la mayoría de votos en la localidad, 402 frente a 1814 obtenidos por los apristas, declara: "El pueblo de Tacna, sentimental y heroico, hoy más que nunca sigue viviendo en mi corazón, precisamente la verificación del proceso electoral con respecto a este departamento, me hace ver que debo dedicarle un mayor acentuado, mi patriótica atención". (La Nación, 12 de noviembre de 1931, p.1) Este anuncio avivaría las fuerzas al comité sanchezcerrista local, para continuar con la labor partidaria.

Para el Congreso Constituyente, resultan ganadores los dos candidatos apristas locales, el nuevo director del diario “La Nación”, Juan Arce Arnao y Gustavo Neuhaus. La oposición reacciona impulsando una tacha sobre ambos. Contra el primero la presenta el Dr. Alfredo Herrera, personero de la UR y por el segundo los señores ex candidatos Sr. Coronel Urdanivia, Bruno Vargas y Carlos Nalvarte, los cuales fundarán "La Liga Patriótica". Se acusa a ambos de no tener residencia y no ser naturales de la localidad. Al final, el JNE resuelve la no procedencia de la tacha y ordena se les otorgue las credenciales (La Nación, 10 de noviembre de 1931, p. 4).

Al conocerse oficialmente el triunfo del Comandante Sánchez Cerro, los simpatizantes locales se manifestaron por las calles de la ciudad, produciéndose algunas escaramuzas el día 8 y 9 de diciembre. El 8 de diciembre, a las 5:30 pm, se dio inicio a una manifestación recorriendo las principales calles de la ciudad, llevando una bandera nacional con el retrato del Comandante Sánchez Cerro. Los hechos violentos se suscitaron al pasar por el local del Partido Aprista, donde algunos manifestantes intentaron arrancar el rótulo que se exhibía en tal local con el nombre del partido, pero fue impedido por la policía. A las 7:30, al detenerse la manifestación en la plaza, el doctor Villa de la Tapia (antiguo director del Diario “La Nación”) pronunció un discurso, recibiendo los aplausos de los manifestantes, siguiendo el recorrido por la calle San Martin, separándose aproximadamente hacia las $8 \mathrm{pm}$. A las 10 de la noche del mismo día, un grupo se aproximó a la puerta del local del partido aprista, dos de ellos arrancaron el rótulo y lo destrozaron en el suelo. Al día siguiente, a las 4:30, tres sujetos intentaron replicar dicho acto, siendo repelidos por los guardias de seguridad. (La Nación, 10 de diciembre de 1931, p.1).

\section{El Comité Departamental de la UR y el Diario "La Verdad"}

En noviembre de 1931, se emprende un proceso de incorporación de los clubes populares en el cuadro orgánico del Partido Unión Revolucionaria (Molinari, 2009 , p. 33) y se consolida la creación de los comités departamentales, como es el caso del Comité Departamental del PUR de Tacna. En 1932, el Comité Departamental del Partido Unión Revolucionaria, como órgano superior en el escalafón a los sub-comités representados por los clubes Sánchez Cerro, tenía como presidente al Señor Felipe Pérez y como secretario al señor Luis Zeballos (La Verdad, 8 marzo 1932, p. 2). En esta etapa, cobra vital importancia el Diario "La Verdad", en cuanto su director Luis Raúl Hernández fue militante de la UR, e incluso se llegó a establecer la secretaría del Partido en el local del diario (el mismo taller donde se editaba "La Nación"). Al respecto, Panty expresa: "Fue un diario noticioso, carente de editoriales, reprodujo artículos de 'El Comercio' de Lima sobre el acontecer nacional. Sirvió de vocero del sanchezcerrismo y fustigó acremente a la oposición aprista a la que calificó de 'secta revolucionaria, antinacional, extranjerizante y comunista' (1998, pp. 88-89). Dicho diario, al coincidir su origen con la promulgación de la "Ley de Emergencia" -que estableció la censura a los medios de la oposición- se arrogó el monopolio de la opinión pública,

\section{Reacciones frente al atentado perpetrado contra Luis M. Sánchez Cerro}

El año de 1932, denominado por Godínez como "El Fatídico Año del Mocho", por Thorndike como «El Año de la Barbarie» y por Kapsoli "El Año del Poron-Mocho" (Kapsoli, 1932, p. 90), se caracterizó precisamente por la violencia política, tanto institucionalizada-de parte de las fuerzas estatales-y por los movimientos considerados como "sediciosos", encabezados por el aprismo. Así como afirma Peter Klaren, el "Perú se precipitó rápidamente hacia el abismo de una guerra civil" a partir de hechos como el atentado al Presidente Luis M. Sánchez 
Cerro, el arresto de Haya de la Torre, el motín naval del puerto del Callao, el levantamiento aprista de Trujillo y las consecuencias fatales (Klaren, 2012, p. 338).

El 7 de marzo, al mediodía, el Presidente Luis M. Sánchez Cerro, ingresa a la parroquia de Miraflores, cuando de pronto se oyen siete disparos, uno impacta en su tórax y el resto hiere a otros asistentes. El perpetrador del atentado era José Melgar, militante aprista, quien fue apresado en su intento de huida (La Verdad, 8 marzo 1932 , p. 2). Este será solo uno de los motivos, que llevaron a una dura represión contra los apristas, respaldada por la Ley de Emergencia, emitida ese mismo año, que establecía la pena de muerte para aquellos "actos de sedición” (Molinari, 2009, p. 52). La apelación a este atentado, sería utilizada como estrategia de legitimación de una serie de actos de violencia física y simbólica, por parte del Estado y los adherentes al partido de gobierno, contra la oposición.

Hubo reacciones de indignación a lo largo del país, por los militantes y simpatizantes del partido de gobierno. Así, en el plano local, se convoca a sesión extraordinaria el 7 de marzo a las 11 y 30 am en el local del "Hotel Vergara", a fin de acercarse a la prefectura y manifestarse contra el atentado al Jefe Supremo del Partido. En tal reunión, un numeroso grupo de militantes del partido y simpatizantes del gobierno "acordaron que una comisión compuesta de varios de los asistentes se acercase a la Prefectura del Departamento y dirigir telegramas al Presidente de la República, el Presidente del Consejo de Ministros y al Presidente del Congreso Constituyente" (La Verdad, 8 marzo 1932, p. 2). Informa el diario:

\footnotetext{
Los elementos que componen la comisión designada, que era un tanto numerosa bajaron por la calle San Martin y Plaza Colón hasta la prefectura, lanzando vivas al Presidente de la República y mueras al aprismo y sus afiliados. El Señor Prefecto Alejandro Saco Arenas recibió en su despacho a la comisión. Hizo uso de la palabra el señor Felipe Pérez, manifestando al señor prefecto la enérgica protesta que expresaban y pedían que así se lo hiciera conocer al Presidente de la República, así como el deseo de que se impusiese la más severa sanción a los culpables. El Señor Prefecto, respondió agradeciendo la manifestación y expresando que telegráficamente comunicara la protesta al Jefe de Estado, lo mismo que la petición de que se castigue duramente a los comprometidos en tan incalificable atentado. [...] (La Verdad, 8 marzo 1932, p. 2).
}

Dichos telegramas fueron firmados por el Presidente, el Secretario del Partido y otras cien personas las cuales -como señala el Prefecto- provenían "de todas esferas sociales" (La Verdad, 10 marzo 1932, p. 2), lo que dejaba entrever, que el apoyo al gobierno y el rechazo al aprismo, era generalizado.

En vista de tal atentado, el Congreso Constituyente presidido por Luis A. Eguiguren otorga facultades excepcionales al Poder Ejecutivo para enfrentar la convulsionada situación. Asimismo, este respondió al telegrama enviado por la comisión urrista por medio de una circular enviada a todos los Prefectos del Sur de la República. (La Verdad, 10 marzo 1932, p. 2). El día 15 de Marzo de 1932, se celebra en la capital una Misa Campaña en la Plaza Bolognesi, donde surge una manifestación hasta la Plaza San Martín. Desde Tacna los sanchezcerristas se adhieren a tal marcha por medio de un telegrama:

[...] En sesión solemne Unión Revolucionaria acordamos adhesiones imponente manifestación realizada en capital en homenaje a nuestro ilustre mandatario Comandante Luis M. Sánchez Cerro, rogámosle hacer llegar conocimiento Señor Presidente los votos sinceros de los verdaderos sanchezcerristas de Tacna que anhelan su pronto restablecimiento firmas.-Junta Directiva Presidente Felipe Pérez V. Secretario Luis Zevallos, Vocal de Turno Víctor A. Lanatta, Vocal J. Abel Chirinos y 115 firmas más. (La Verdad, 10 marzo 1932, p. 2)

Este tipo de comunicados, fueron emitidos por los comités urristas de todo el país, algunos denotando un discurso mucho más violento, sobre todo aquellos que eran emitidos por los propios comités, en contraste con los emitidos por autoridades políticas (Molinari, 2009, pp. 78-86). Este autor señaló, que dicho atentado fue instrumentalizado políticamente por el PUR, respecto a "la movilización de sus bases y de los sectores populares sobre los que influían, indignados por tal acontecimiento" (2009, p. 87) haciéndolos partícipes del conflicto político, rasgos que reforzarían el liderazgo carismático del caudillo, en una relación de "intersubjetividad autoritaria entre Sánchez Cerro y las multitudes populares y de sectores medios que conformaban las bases fundamentales del movimiento sanchezcerrista" (Molinari, 2009, p. 90). Como vemos, este atentado será incorporado al discurso urrista, constituido hasta entonces por la apelación a la "Revolución de Arequipa" -representado como una suerte de desagravio nacionaly que buscaría legitimar los actos represivos del gobierno, así como generalizar el rechazo al aprismo.

\section{Creación del Comité del Partido Unión Revolucio- naria de Tarata}

Una de las consecuencias de la crisis del 29 fue la desocupación. En Tacna, por el año 1931, habían 400 desocupados, registrados por la Junta Pro-desocupados de 
Tacna, representando el 12\% de una población masculina de 3300 residentes, cifra que no varió hacia 1935 , como se observa en el Censo de 1935, pues existían 302 desocupados, representando el 8,5\% de la población masculina de 3550 residentes. Para enfrentar esta situación, el "Comité pro-desocupados de Tacna" (creado en julio de 1931) impulsó algunas obras para dar trabajo a los desocupados, empezando con la reparación de la carretera Tacna-Tarata (Panty, 2001, pp. 16-17).

Aprovechando la condición de ser partido oficialista, el Comité Departamental hace un llamado el 22 de marzo de 1932, a los desocupados afiliados, para tenerlos presente en la distribución del trabajo (La Verdad, 22 marzo 1932, p. 3), sin embargo, se considera que realmente el objetivo era la afiliación de nuevos miembros desocupados, por motivos de necesidad y la posición privilegiada del partido. Muchos de los desocupados inscritos fueron enviados a trabajar en la construcción de caminos de Tarata a Ancomarca, a cargo del señor Víctor Lanatta, a la par se inician las gestiones para la creación del Comité de la Unión Revolucionaria en dicha localidad, constituyéndose oficialmente el 11 de abril, teniendo como Presidente al señor Alfredo Arce Arnao (La Verdad, 15 abril 1932, p. 3). El diario "La Verdad", a la par que saluda la creación del novísimo comité, arremete violentamentecontra el aprismo señalando:

[...] Tiempo ya era de que en esa importante provincia, los ciudadanos se dieran cuenta de que militar en la filas del aprismo es una acción vergonzosa y deshonrante para todo peruano y que ingresar al Partido nacionalista por excelencia de ideales claros y benéficos para la organización de nuestra nacionalidad es una obligación ineludible que todo patriota debe cumplir inmediatamente ahora que el país reclama, más que en ningún otro momento de su historia, la cooperación decidida de todos los hombres de acción (La Verdad, 16 abril 1932, p. 3).

Mostrando como un acto reivindicativo la creación de dicho partido, para borrar la huella del aprismo en dicho sector y esperando se generalice en toda la localidad; apelando nuevamente al sentimiento patriótico arraigado en los tacneños y que el partido de gobierno dice encarnar; por ende el apoyo 0 afiliación, se presenta como una cuestión de carácter moral.

\section{La visita de un miembro del Comité Directivo de Lima}

Con el aumento de militancia, se establece la Secretaria del Partido en los altos de la imprenta "La Verdad", asimismo, se completó el directorio del partido, que hasta entonces tenía como directiva a Felipe Pérez como Presidente, Luis Zevallos como Secretario, como Vocales a Víctor Lanatta y J. Abel Chirinos. La lista de los elegidos en la sesión del 17 de abril son los siguientes: Vicepresidente Luis Díaz, Tesorero el señor José Valdivia y defensor de asuntos obreros el señor don Lorenzo Osorio (La Verdad, 17 abril 1932, p. 2). En la misma reunión, el Comandante del Resguardo, Enrique Caballero expuso la necesidad de que los mejores cuadros del sanchezcerrismo local ocupan puestos públicos, especialmente en la aduana y reguardo, pues "la mayoría de empleados de estas secciones eran enemigos del Gobierno del Coronel Sánchez Cerro (La Verdad, 17 abril 1932, p. 2)", haciendo referencia principalmente a los trabajadores de filiación aprista.

El día 16 de junio de 1932, se cita a los militantes con motivo de la visita del señor Carlos Ramírez Alzamora, miembro del Comité Directivo de la Central de Lima. La cita era en la "Quinta Cural", ubicada en la calle A. Blondell. Al día siguiente se publica una reseña de tal encuentro, en la cual deja constancia de la asistencia de más de 250 miembros del partido junto con autoridades como el Prefecto del Departamento, el subprefecto, el intendente de policía de la provincia del Cercado y el Secretario del prefecto, así como también del Director del diario, el teniente coronel don Alejandro Ruiz Bravo, Comandante de Armas y primer jefe del Glorioso Regimiento "Húsares de Junín $\mathrm{N}^{\circ} 1$ y el acompañamiento de la Banda Municipal; informando de esta manera:

[...] A la hora final, ofreció la manifestación el señor Felipe Pérez, presidente del Partido en esta ciudad, que en conceptuosas frases puso de manifiesto la sencillez del homenaje pero la sinceridad que lo inspiraba, e hizo alusión a los ideales que la "Unión Revolucionaria" trataba de encarnar en el espíritu de todas las clases sociales, para el bien de la Patria y terminó brindando por la prosperidad del agasajado [...] El lunch de referencia ha servido para apreciar el incremento que cada día viene tomando el Partido "Unión Revolucionaria" debido a la fe con que realizan una alturada labor de propaganda sus principales miembros, que se hallan poseídos del convencimiento del papel que ha de desempeñar en la hora actual de nuestra nacionalidad" (La Verdad, 17 abril 1932, p. 2).

De esta forma, el crecimiento del partido al que hace referencia el diario, se puede explicar a partir de que la presencia del sanchezcerrismo no solo se encontraba en las instituciones de gobierno (y el sector público en general), sino en las instituciones militares-policiales (posición que podría ser aprovechada, sobretodo en un contexto de conflicto político) y en la prensa (monopoli- 
zando la opinión pública, respaldado por la Ley de Emergencia), configurándose el escenario propicio para las actividades proselitistas.

\section{Aniversario de la "Revolución de Arequipa"}

A pocos días de conmemorarse la "gesta" del Comandante Sánchez Cerro, el Partido por medio del diario, convoca a todos los afiliados y simpatizantes a que se inscriban en la Secretaria del Partido para la fiesta conmemorativa, que en palabras de su secretario era la "fecha en que el gesto de nuestro glorioso jefe cayó la tiranía del oncenio" (La Verdad, 17 agosto 1932, p. 2).

Y sobre los festejos locales, en consonancia con "los varios miles" que se manifestaron en Lima, el diario "La Verdad” reseña: Se inició a las 3 pm con un recorrido por las principales calles portando estandartes alusivos a la fecha, hasta llegar a la Plaza Colón, donde los manifestantes se situaron al frente de la prefectura, de donde salieron por el balcón el Prefecto, el Subprefecto, el Capitán Comisario y algunas otras personas más. Contó con un discurso a cargo del Señor Ricardo Téllez y el Señor Eulogio Cavallero Comandante del resguardo de Tacna. Después el desfile, recorrió unas calles más, llegando a la Quinta Curol" para un banquete (La Verdad, 24 agosto 1932, p. 2).

En la misma edición, se reproducen las palabras de Eulogio Caballero, Comandante del resguardo de Tacna, manifestadas en tal ocasión:

[...] Sánchez Cerro es la encarnación del valor consciente, que es rectitud, nobleza, sinceridad y acción, el valor es siempre más elevado representante de la personalidad humana; su figura se destaca con reflejos de gloria, en las tres tentativas para derrocar al tirano, derramando su preciosa sangre en el altar de la patria [... ] (La Verdad, 24 agosto 1932.p.3).

Y el discurso pronunciado por un partidario llamado Jorge Espinoza en la cena conmemorativa:

"Ciudadanía, un día como hoy, el 22 de agosto de 1932, el entonces comandante Luis M. Sánchez Cerro, jefe de las fuerzas de Arequipa, se levantó en armas interpretando el sentimiento unánime de los pueblos del Perú, que se sentían agobiados por la nefasta tiranía de Leguía y esta revolución triunfa desde Tacna hasta Tumbes y desde la Costa hasta las Montañas y triunfa porque es la justicia que se impuso y porque es el sentir de los heroicos hijos de Grau. [...] Ciudadanos el 22 de agosto que la historia del Perú en sus páginas recordará el nombre de Sánchez Cerro, del militar héroe, de nuestro actual Presidente, que con su espada supo triunfar y que elegido por la voluntad popular, nos liberó también de la plaga Aprista que venía a cegar nuestros espíritus [... ]" (La Verdad, 24 agosto 1932, p. 3).

Ambos discursos, confirman la condición de Sánchez Cerro, como "mesías político" (Molinari, 2009, p. 20), dentro del imaginario popular sanchezcerista, sobre la cual se articula toda la actividad del PUR; ocupando la denominada "Revolución de Arequipa", el lugar de mito fundacional, el cual era apelado constantemente, a fin de presentar dicho suceso como "prueba histórica" de las cualidades "excepcionales" del líder, buscando reforzar la construcción de la figura del "héroe".

\section{El comité departamental de la UR y la colecta "Pro víctimas militares de los sucesos de Trujillo"}

Jorge Basadre, en su monumental obra "Historia de la República" afirma que "En el Perú de 1932 hubo, por ambos bandos contendientes, un vasto derramar de sangre y de encono que anticipa el de la guerra española de 1936 a 1939" (2014, p. 211). En efecto, luego de la sublevación de miembros de la Marina de Guerra en el Callao -hecho atribuido al PAP-, en la mañana del 7 de julio de 1932, en Trujillo, insurrectos toman el cuartel de la ciudad para expandirse hacia el Puerto de Salaverry y a la Costa norte, Huamachuco, Otuzco y Santiago de Chuco en la Sierra. El gobierno responde con tropas enviadas desde la capital y desde Lambayeque. Los dirigentes apristas escaparon, se asesinaron a los jefes y oficiales de la guarnición de Trujillo e incluso se encontraron cadáveres mutilados y si bien las cifras son discutibles, se hablaba de hasta 5000 apristas fallecidos producto de la represión de las Fuerzas Armadas (Pease, 1993, p. 163)

En el plano local, se instala la "Junta Departamental de Fondos pro-víctimas militares de los sucesos de Trujillo", que había acordado "invitar a todas las personas de buena voluntad", para la colecta "Pro-víctimas militares de los sucesos de Trujillo", donación que se haría entrega en "la oficina de la Caja de Depósito y Consignaciones, Departamento de Recaudaciones", instancia que a su vez entregaría a cada erogante la constancia de su óbolo, que se recibirían hasta el 31 de dicho mes y se publicaría la lista en el diario (La Verdad, 21 de agosto de 1932, p. 3).

Al no obtener los resultados esperados con la colecta en la ciudad, el Comité Departamental del Partido Unión Revolucionaria, llamó a militantes y simpatizantes de los comités distritales y provinciales (Tarata, Ticaco, Candarave, Curibaya, Huanuara, Ilabaya, Locumba) para aportar. La Verdad reproduce el siguiente telegrama: 
[...] Comité "Unión Revolucionaria" ésta, acordó lanzar iniciativa a demás comités distritales y provinciales departamento, que partido patrocine entre sus afiliados y simpatizantes erogación pro-armamento nacional.Punto-Esperamos de su patriotismo activa gestión fin contribución con máximo esfuerzo defensa patria que es el más grande ideal de nuestro partido. [... ] (La Verdad, 1 octubre 1932,p.3)

La poca acogida de esta campaña, evidenciaría, que a pesar de que el PUR en la localidad había incrementado considerablemente sus bases o comités y por ende el número de afiliados (aunado a las relaciones directas con las instituciones del gobierno central, local, militarpolicial y la prensa escrita), seguían representando un número inferior respecto a la tendencia aprista.

\section{El primer aniversario del gobierno constitucional de Sánchez Cerro}

En el aniversario de la victoria electoral del Comandante Luis Miguel Sánchez Cerro, a pesar de haber perdido en la localidad, se pudo ver con el paso del tiempo el crecimiento del partido y la proliferación de comités. Muestra de ello, fue la realización de actividades de todo tipo, con la participación de las instituciones, los militantes y la población en general. El programa de esta fecha solemne se inició con el izamiento del pabellón con los honores de las fuerzas de policía, un almuerzo en el Parque Municipal, una función de "cinema" en el Teatro Municipal, auspiciado por el empresario de teatro apellidado Batts, quien donó 500 entradas para que se distribuyan entre los miembros del partido y a las 9 se dio inicio el baile popular en el mercado (La Verdad, 10 diciembre 1932, p. 2). Respecto al éxito de dicha actividad, el diario señala:

"En suma los diversos actos que mostramos ligeramente indicarían de manera elocuente la adhesión de los pobladores de Tacna a la política del General Sánchez Cerro y al reconocimiento que por su actitud levantada y patriótica ha sabido imponer a la ciudadanía (La Verdad, 10 diciembre 1932,p. 2).

Por ello, es posible inferir que hasta ese momento, el partido contaba con un aproximado de 500 miembros, duplicando lo constatado a mitad del mismo año.

\section{Reacciones frente al asesinato de Sánchez Cerro}

El domingo 30 de abril de 1933, en el Hipódromo de Santa Beatriz, se efectuó un desfile de treinta mil movilizables, acto al que acudió el Presidente de la República, presenciándolo desde la tribuna oficial, hasta aproximadamente una de la tarde, donde sale entre aplausos de la multitud en un automóvil descubierto, De pronto, un individuo se abalanzó a la parte trasera del auto y acribilló por la espalda a Sanchez Cerro, con tres disparos (según peritaje médico). Del mismo modo, el agresor cayó muerto por las balas de uno de los "edecanes". El auto aceleró con dirección al Hospital Italiano, donde tras agonizar, falleció a la una y diez de la tarde (Basadre, 2014, pp. 54-55).

El 1 de mayo del mismo año, el diario informa sobre el traslado de los restos del General Sánchez Cerro a la Iglesia del Sagrario, acompañado de gran cantidad de público, "el dolor popular", identificando al ciudadano Abelardo Mendoza Leiva, como el asesino del Presidente, "el aprocomunista" -como lo denominan- quien "habría sido detenido en varias ocasiones por atentados en contra del orden social establecido en el país" (La Verdad, 1 de Mayo de 1933, p. 2).

Las honras fúnebres programadas en la Capilla Espíritu Santo, el primero de mayo, se postergó por acuerdo del Consejo de Ministros hasta el día siguiente "con el objeto de dar lugar a que todo el pueblo visite la cámara mortuoria”, llevándose a cabo a las 10 am del 2 de mayo (LaVerdad, 1 mayo 1933, p. 3).

Se envían telegramas por parte de autoridades locales como el Alcalde de Tacna, Luis Gregorio Zevallos Cruz a Paolo Ernesto Sánchez Cerro (hermano del General Luis Miguel Sánchez Cerro), transmitiéndole sus condolencias y del Alcalde de Candarave, como informa el diario: "El Alcalde de Candarave expresa el sentimiento y la consternación de un pueblo por el horrendo crimen cometido en la persona del General Sánchez Cerro" y señalando que se realizará la misa a las 9.00 am en la Capilla de la localidad (La Verdad, 1 mayo 1933, p. 3). En consonancia, con la romería hacia al recinto mortuorio que reunió a más de 50000 personas y las movilizaciones en todo el país (La Verdad, 4 mayo 1933, p. 2), dicha municipalidad manda a oficiar una misa el 6 de Mayo, a la que asisten miembros del Concejo Distrital, Gobernador del distrito, miembros de la Unión Revolucionaria, centros escolares, los movilizables del Ejército, el Club de Artesanos y numerosa población, como informa el diario (La Verdad, 7 de mayo de 1933, p. 3).

Lo mismo sucedió en la provincia de Tarata, como se informa por medio de un telegrama. Asistieron autoridades, centros escolares de ambos sexos, numerosos pueblos, movilizables, pobladores de la capital de la provincia, así como del distrito Ticaco, Tarucachi, con el acompañamiento de una "Banda de Guerra" (La Verdad, 7 mayo 1933, p. 2). 
Desde este momento, es designado jefe de gobierno Oscar R. Benavides y el comité central de la UR se reúne con el mismo para hablar sobre el futuro del país y especialmente del partido (La Verdad, 19 mayo 1933, p. 2). El 25 de mayo, en el Teatro Municipal se exhibe una película sobre los "imponentes funerales del Presidente General Luis M. Sánchez Cerro", siendo Tacna la primera provincia donde se exhibe (La Verdad, 25 mayo 1933, p. 2).

\section{La escisión dentro de la UR y el Comité Departa- mental}

Contrario a los primeros dos años, las actividades que duraban todo el día organizadas por el Comité Departamental de la Unión Revolucionaria y las autoridades locales, tras la muerte del Gral. Sánchez Cerro, no se observa movimiento del Comité local ni mayores actividades por la fecha, más allá del embanderamiento general decretado por el aún alcalde Luis Zeballos C, un tímido editorial agrega "la gratitud del país con el ciudadano ilustre que le dio la libertad que había sido eclipsada durante más de una década" y una ceremonia/conferencia realizada por el Colegio Nacional de Varones (La Verdad, 22 agosto 1933, p. 1), pudiendo observarse el ingreso a un periodo de decadencia del sanchezcerrismo..

Es posible que la causa de la poca presencia del Comité Departamental sea la ruptura dentro del PUR, liderada por Clemente Revilla quien forma el "Partido Nacionalista” que automáticamente se adhirió al régimen, e incluso un minúsculo grupo se afilia al Apra (Molinari, 2009, p. 107). Lo cual implicaría, la aplicación de un reingeniería, dentro del partido, si se plantea sobrevivir ante un escenario desfavorable, sobretodo, por la pérdida del caudillo, eje articulador de las diversas fuerzas operantes al interior de la UR.

De esta manera, ante un comunicado enviado por el Comité Departamental del Partido Aprista Peruano al Presidente pidiendo la destitución del Comandante en Jefe de la Guardia Civil y Policía de Tacna, se remite un comunicado a nombre del "pueblo de Tacna" donde -entre aquellos que firman el comunicado-se puede observar a Víctor A. Lanatta y Felipe Pérez Vélez, miembros del consejo directivo del Comité Local de la UR, pero en esta oportunidad, mostrándose como simples ciudadanos.

[...] No es pues el pueblo de Tacna el que reclama el nombramiento del Comandante Matto, es solamente el grupo aprista, que quiere imponer su voluntad en todos los sectores del territorio y siembra desconfianza, que insta a la anarquía y sobretodo quiere la disociación, construyendo en las personas el odio a todo lo que está legalmente constituido. Sírvase Ud. Señor Presidente tomar el memorial aprista únicamente como de un grupo, pero no como una manifestación del pueblo de Tacna. [...] (La Verdad, 30 noviembre 1933, p. 3).

El único comunicado publicado por el Partido en ese año, luego de la muerte del caudillo, es aquel que informa el traslado de su Secretaría que se ubicaba en los altos del Diario "La Verdad" hacia la Calle 2 de Mayo No 464 (La Verdad, 1 diciembre 1933, p. 2). Si en 1933 la actividad partidaria y su presencia en su otrora vocero "La Verdad" fue mínima, en 1934 es casi imperceptible.

Desde el 12 de enero hasta el 1 de febrero de 1934 , se anuncia en un pequeño comunicado citando por disposición de la Secretaría de Organización del Comité Central a los afiliados para la reinscripción y cambio de carnets en su nuevo local de la calle 2 de Mayo $\mathrm{N}^{0} 464$. Al conmemorarse el primer año del asesinato del Presidente Sánchez Cerro, el Comité Provincial del Partido, invita a sus correligionarios y "personas piadosas" a la Misa de Réquiem a celebrarse el día 30 de abril en la Vicaría, a las 3:30 pm con motivo del aniversario de su fallecimiento (La Verdad, 29 abril 1934, p. 3).

De este modo, a la par de los sucesos nacionales, pugnas dentro del partido, escisiones, acuerdo y posterior persecución de 0scar Benavides al partido, se produce un interregno que generará un desequilibrio, pero que cerrará esta etapa sanchezcerrista y dará inicio a la denominada etapa "fascista" al mando de Luis A. Flores, quienes mantendrán el culto al caudillo, pero esta vez con una ideología estructurada, con una visión corporativista del estado y la nación, tomando como ejemplo la experiencia de la Italia de Mussolini, lo que José López Soria, denominaría "fascismo popular" (López, 1981, p. 25).

\section{CONCLUSIONES}

Si bien en Tacna, no llegó a masificarse el apoyo a Luis M. Sánchez Cerro, como sí lo hizo el aprismo, un importante sector de la población se adhirió a su proyecto desde la denominada "Revolución de Arequipa", enfrentándose en un inicio -al igual que ocurría en otras ciudades- por medio de la prensa contra sus opositores políticos, principalmente apristas. De las diversas declaraciones rescatadas de los diarios sanchezcerristas de la época, se infiere que gran parte del apoyo a la candidatura de Sánchez Cerro estaba revestida de un carácter mesiánico, por cuanto Leguía era considerado un traidor por la entrega de Arica, sería Sánchez Cerro, quien devolvería la dignidad al pueblo tacneño que pedía con urgencia atender sus necesidades, anhelo que no fue materializado, producto de la atención del gobierno de los conflictos dentro (el 
estallido de movimientos insurreccionales) y fuera (conflicto con Colombia) del país.

A partir de la emisión de la denominada "Ley de Emergencia", la facción sanchezcerrista, producto de la censura a la primera fuerza política de la localidad que fue el aprismo, quedó como la única fuerza política con libertad de actuación, lo que facilitó la actividad propagandística, al monopolizar la opinión pública, especialmente por medio del diario "La Verdad"; siendo respaldados por la presencia de afiliados y simpatizantes en la administración pública, generándose un escenario propicio para el proselitismo, visualizándose algún crecimiento de afiliados; sin embargo, se mantuvieron en segundo lugar, respecto a la tendencia aprista.

La identificación con el proyecto populistaconservador del sanchezcerrismo, podría haber sido meramente coyuntural, pues tras el asesinato de Luis M. Sánchez Cerro -y en consonancia con los propios cismas y problemas internos del PUR a nivel nacional-se evidenció una inactividad nunca vista desde el surgimiento de dicha fuerza política en la localidad.

\section{REFERENCIAS BIBLIOGRÁFICAS}

Basadre, J. (2014). Historia de la República del Perú. Lima: Producciones Cantabria.

Drinot, P. (2001). El comité sanchezcerrista de Magdalena del Mar, un ensayo de microhistoria política. Revista del Archivo General N ${ }^{\circ} 23,333-353$.

Gonzales, E. (1994). La derecha latinoamericana en busca de un modelo fascista: la limitada influencia del falangismo en el Perú (19361945). Revista Complutense de Historia de América, núm. 20: 229-255.

Kapsoli, W. (2015). El año del "Porón-Mocho" (Perú: 1932). Studia Histórica, Vol. 33, 87-107.

Klaren, P. F.(2012). Nación y Sociedad en la Historia del Perú. Lima: Instituto de Estudios Peruanos.

Lopez, J. I. (1981). El Pensamiento Fascista (19301945). Lima: Mosca Azul Editores.
McCombs, M. E. (1994). "La comunicación de masas en las campañas políticas: información, gratificacióny persuasión. En Sociología de la Comunicación de Masas (Vol III. Propaganda politica y opinión pública). Barcelona: Ediciones G. Gili S.A.

Molinari, T. (2006). El Partido Unión Revolucionariay su proyecto Totalitario Fascista 1933-1936. Investigaciones Sociales Año X, No 16: 321-346.

Molinari, T. (2009). El fascismo en el Perú: La Unión Revolucionaria 1931-1936. Lima: Fondo Editorial de la Facultad de Ciencias Sociales- UNMSM.

Morán, D. y Aguirre, M. (2008). La prensa y el discurso político en la historia peruana: Algunas consideraciones teóricas y metodológicas. Investigaciones Sociales Año XII, 229-248.

Mussolini, B. (1940). Espíritu de la revolución fascista: antología de los escritos y discursos. Bilbao: Vizcaína.

Panty, 0. (1998). Historia de la prensa escrita en Tacna. Tacna: Universidad Jorge Basadre Grohmann.

Panty, 0. (2001). Función orientadora en la prensa escrita. Tacna: Educa.

Payne, S. (2005). El Fascismo. Madrid: Alianza Editorial.

Pease, F. (1993). Perú Hombre e Historia: La República III. Lima: EDUBANCO.

Savarino, F. (2005). La ideología del Fascismo, entre pasado y presente. Diálogos entre la Historia social y la Historia cultural, 253-272.

Strauss, L. (1965). Natural Right and History. Chicago: The University of Chicago Press.

FUENTES PRIMARIAS (ARCHIVO REGIONAL DE TACNA)

Diario "La Nación” (1931)

Diario "LaVerdad" (1932-1934) 